\title{
Embedded fuzzy logic controller for positive and negative pressure control in pneumatic soft robots
}

Conference or Workshop Item

Accepted Version

Oguntosin, V., Nasuto, S. and Hayashi, Y. (2018) Embedded fuzzy logic controller for positive and negative pressure control in pneumatic soft robots. In: 2017 UKSim-AMSS 19th International Conference on Modelling \& Simulation, 5-7 April 2017, Cambridge, U.K.. Available at http://centaur.reading.ac.uk/73376/

It is advisable to refer to the publisher's version if you intend to cite from the work. See Guidance on citing.

Published version at: https://doi.org/10.1109/UKSim.2017.41

All outputs in CentAUR are protected by Intellectual Property Rights law, including copyright law. Copyright and IPR is retained by the creators or other copyright holders. Terms and conditions for use of this material are defined in 
the End User Agreement.

www.reading.ac.uk/centaur

\section{CentAUR}

Central Archive at the University of Reading

Reading's research outputs online 


\title{
Embedded Fuzzy Logic Controller for Positive and Negative Pressure Control in Pneumatic Soft Robots
}

\author{
Victoria Oguntosin ${ }^{1}$, Slawomir J. Nasuto ${ }^{1}$ and Yoshikatsu Hayashi ${ }^{1}$ \\ ${ }^{1}$ Biomedical Engineering Section, School of Biological Sciences, University of Reading, UK \\ \{v.w.oguntosin@pgr.s.j.nasuto@,y.hayashi@\}reading.ac.uk
}

\begin{abstract}
A key challenge in soft robotics is controlling the large deformation experienced as a result of high compliance nature of soft robots. In this work, a software control strategy for regulating the amount of internal positive and negative air pressure inside pneumatic soft robots is presented. Since the air pressure has a direct effect on the amount of deformation, the position of the robot is controlled. Pressure control was implemented with a fuzzy logic controller, which is described with its performance shown. The approach can be integrated into any specified soft robotic actuator requiring pneumatic actuation e.g. bending, triangular and muscle actuators.
\end{abstract}

Keywords- Soft robots; Fuzzy logic control; Positive pressure; Negative pressure; Multichannel actuator

\section{INTRODUCTION}

Soft pneumatic actuators are able to exhibit twisting, bending [1], angular change [2] and rotary movements [3], [4] with the aid of compressed air. These movements are achieved by the variation of air pressure inside the actuator. The precise control of soft robots is considerably challenging because they possess infinite degrees of freedom as they navigate based on deformation of their structure [5]. Through compliance and associated benefits such as safety and soft interaction offered by soft robots, they are more difficult to control when compared with rigid manipulators [6].

Furthermore, pneumatic systems are known to generally suffer from nonlinearity due to air compressibility. They are also difficult to control since identifying a precise system model is cumbersome due to unknown disturbances [7]. Previous work on methods of driving soft actuators is with the use on/off solenoid valves [8], [9], syringes [1] and fluidic drive cylinders [10] to control the flow of pressurized air to the actuators. The use of cylinders is superior to on/off solenoid valves since it enables precise analogue control of airflow into and out soft robots [10].

The effectiveness of pneumatic pressure controllers has been confirmed through step response experiments, both for PWM-controlled on/off valves [11] as well as for different types of servo valves [12], [13]. A feedback control loop with an angle filter was implemented for a fiber reinforced soft bending actuator [14]. Using information about the measured input air pressure from a pressure sensor, the angle filter of the feedback loop estimated the required soft actuator bending angle.
A soft pneumatic system that requires the multiplexing of many fluids, ranges of pressure and changes in flow rate has been implemented [15], the elasticity of silicone rubber was used as valves to open and close air channels. The mode of operation is through the application of a force by piezoelectric actuators that pinches and releases a fluidic channel made of PDMS in order to close or open it at a precise location. The system utilized elastomeric valves that open and close using piezoelectric actuators of a commercial Braille display. This Braille display had 64 individually addressable pins and therefore controlled 32 expandable outputs.

The main contribution of this paper is to present and demonstrate the effectiveness of an embedded fuzzy logic controller for pneumatic soft robots. Fuzzy logic was first used by Lotfi Zadeh [16] and has since been applied for a variety of practical and commercial applications such as in the operation and programming of temperature control of a shower head [17], air conditioners, washing machine and rice cookers [18]. A fuzzy controller optimized by genetic algorithm to control pressure and level of drum boilers has also been designed [19] which had the overall effect of reducing both the settling and rise time. A neural-network based learning algorithm [20] which can self adjust weights of fuzzy rules has been implemented to improve timing parameters of the controller. Fuzzy logic controllers are a way of executing closed loop control and is more intuitive, following more closely with the way a human would control the system. Fuzzy logic is an algebra and similar to boolean logic, while Boolean logic defines two only states (true and false), conditions exist in the continuum between true and false for a fuzzy logic system.

\section{Methodology}

The key elements that constitute the control system of any robot (hard or soft) are:

1) an actuator to physically move the robot;

2) a mechanical transmission to connect the actuator to the robot;

3) a sensor to measure the position of the robot;

4) power electronics to provide electrical current to the actuator; and

5) control algorithm that computes an error signal and sends an appropriate signal to the power electronics. 
The actuator is always the device that can convert a control signal to velocity and force. In the case of pneumatically powered soft actuators, the actuator generates motion (bending, linear and rotary) using the force exerted by compressed air. Pneumatic actuators require the use of hoses, valves and an air source. Soft pneumatic actuators carry their mechanical transmission in their bodies. Internal and external sensors are used to measure the position and/or deformation of soft robots. Power electronics is used to provide current to the electrical components such as the air source and valves. Control algorithms to guide the soft robot to a desired position by sending an appropriate signal to the power electronics is required. An electro-pneumatic hardware system for actuating pneumatic soft robots has been described [21]; this paper builds on the work by detailing the software control strategy employed.

The main aim of this system is to regulate the pressure of compressed air from a pressure source such that a desired output pressure is directed to actuate the robot. The use of an electro-pneumatic regulator and air compressor for this purpose would be expensive. Furthermore, the use of mechanical systems such as cylinders, piston pump would be cumbersome and make the system bulky. For driving soft robot with a compact arrangement, solenoid valves which act as control switches for the flow of air into and out of the actuators are used. Two valves are required for one actuator: the first for inflation (inlet solenoid valve) while the other is for deflation (exhaust solenoid valve). This is a low pressure controller system that operates between about 0 to \pm 5 psi.

The movement and deformation experienced by softbodied actuators is reliant on effective control of airflow into and out of the actuator. This is controlled by on/off solenoid valves that incorporate 2-way/1-position. The electro-pneumatic system is composed of an embedded electronic control on a Printed Circuit board (PCB) and a compact structure to house the pneumatic system so that portability is ensured. $6 \mathrm{~V}$ solenoid valve having a power rating of $2.25 \mathrm{~W}$, a maximum operating pressure of $350 \mathrm{mmHg}$; exhaust speed of $4 \mathrm{~s}$ and leakage of $3 \mathrm{mmHg} / \mathrm{minute}$ was used. These solenoid valves are light-weighted and compact so as to be suitably integrated. In the case of a high pressure application, high pressure valves would be needed - this would be achieved by either operating two solenoid valves in parallel as one logical valve or simply using valves with higher operating pressure.

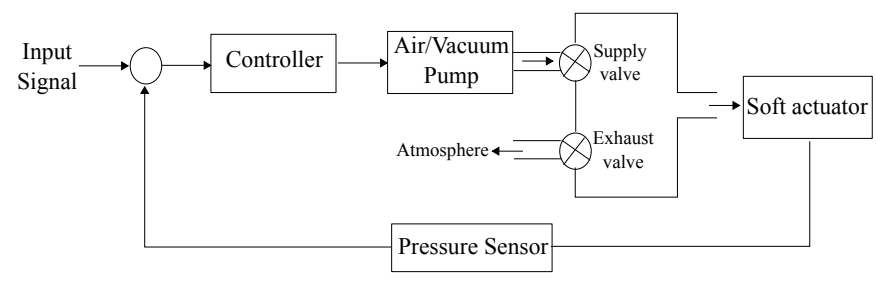

Fig. 1: Block diagram of soft actuator pressure controller.
Fig. 1 shows the block diagram of the electro-pneumatic setup to actuate soft robots: an input signal sets a desired pressure; the controller receives current air pressure in voltage units from the actuator by an Analogue-to-Digital Converter (ADC) interface. When the input signal rises, compressed air from a pressure source passes through the inlet solenoid valve and changes to output pressure. The exhaust valve deflates the soft robot - this is essential to create a frequency at which a soft robot would inflate and deflate in order to create a specified rhythm of motion. The current pressure in the actuator is fed back via a pressure sensor, pressure corrections then occur to produce an output pressure that is equal to the set pressure. Pressure regulation is required to control the motion of the soft robot and most importantly, prevent failure of silicone rubber due to overinflation.

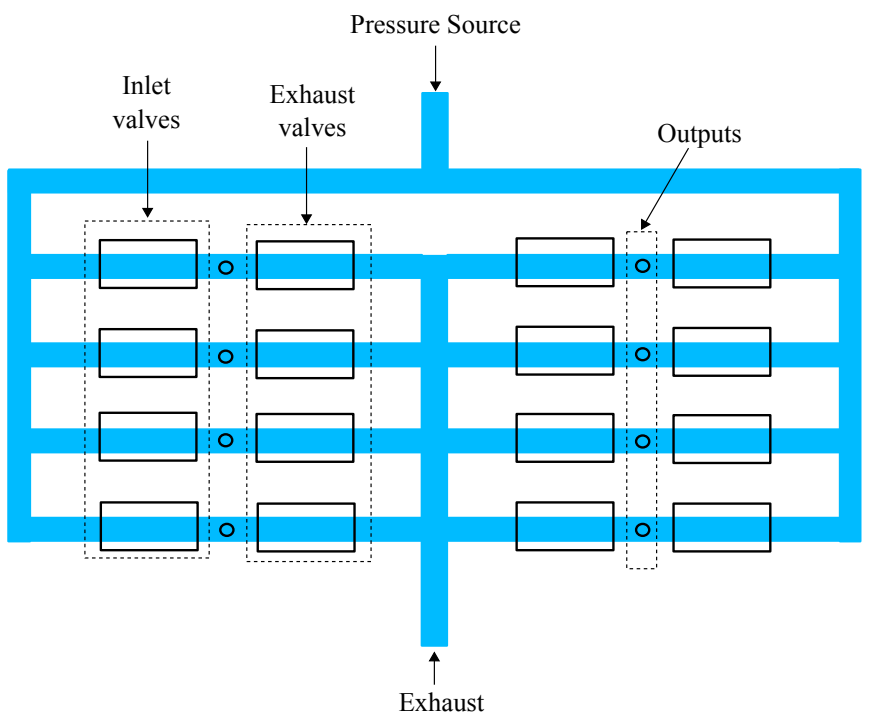

Fig. 2: Arrangement showing network of valves using a single pressure source but feeding 8 outputs independently, Number of valves required is 2 $\times$ number of outputs.

A Pulse Width Modulated (PWM) air pump controls output pressure inside the actuator integrated with a pressure sensor. Pressure is controlled by varying the length of the duty cycle of the DC air pump. In this case, a duty cycle has a maximum length of $10 \mathrm{~ms}$. In order to write the duty cycle duration to control an air pump, a DAC (Digital-to Analogue Converter) is required which can be implemented by using a DAC circuit or transistor arrangement. The output pressure into the soft actuator is also dependent on pressure difference, power supply ratings of valves and pump. For the inlet solenoid valve, pressure difference is given by a constant supply pressure and the actual pressure in the actuator. For the exhaust solenoid valve, pressure difference is between internal air pressure in the soft robot and atmospheric pressure.

More than one separate individual units of a multichannel soft robot can be controlled using this arrangement. This is achieved by dividing the air coming from the pump and 


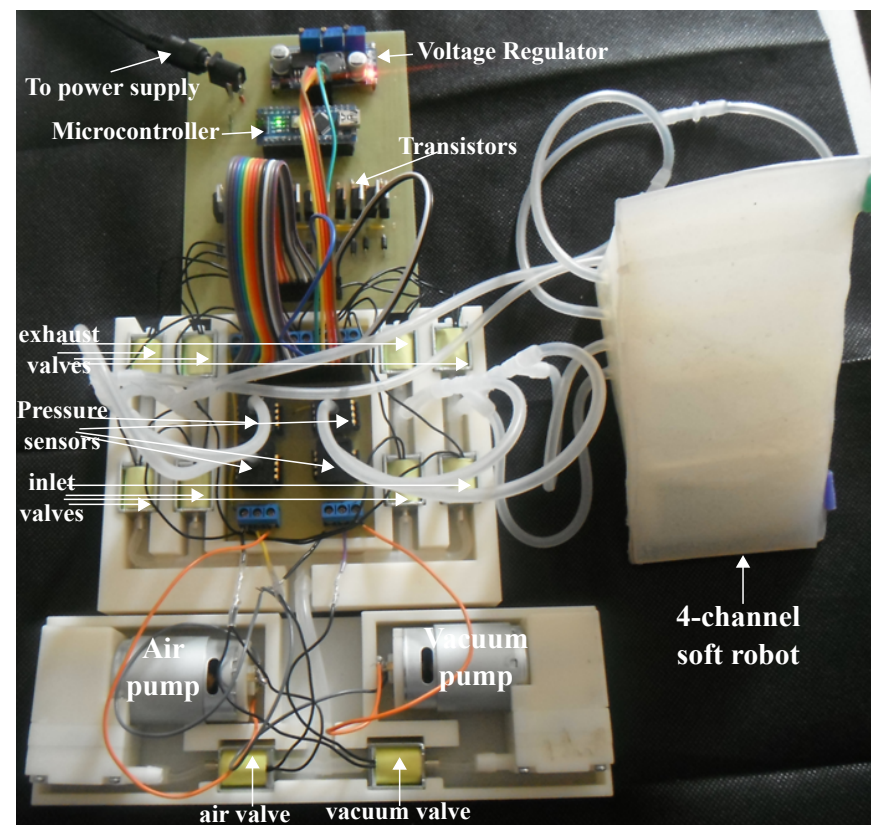

Fig. 3: Implementation of hardware control system for a 4-channel sof robot requiring 8 valves for independent control of air pressure inside each air channel. Positive and negative air sources are combined and then divided into four air channels to regulate air pressure inside individual channels.

using two valves for each air channel; pressure and exhaust can be connected to one or more soft robot at a time. Using separate pressure control for each of the individual air channels illustrated in Fig. 2 and 3 will tend to reduce failure of the soft robot through inflation and increase the obtainable range of motion. Each of the four air channels constituting wedge-shaped soft modules was controlled using this procedure.

\section{Controller Design}

Control systems can either be open loop or closed loop. An open-loop control system implements no feedback path providing information about the current state of the system. Open loop control uses wait-delay periods and would not require the use of sensors. The more advanced closed loop control system uses a sensor to measure the current state variable and uses this information as feedback to check if the desired value has become equal to the current value. Closed loop control systems can be implemented as bangbang, PID or fuzzy logic controllers. A portable and wearable control system implemented with a bang-bang controller for actuating the Bowden cable of a soft robot using a microcontroller system has been demonstrated [22]. A bang-bang controller unfortunately suffers from large errors or very slow response times. To make a faster and more accurate system, a Proportional-Integral-Derivative (PID) controller from linear control theory is often used. A PID controller uses an error signal calculated by the difference between the current and desired state to generate a control input signal which is then fed into the electrical actuator with a pace controlled a real-time clock. The design parameters for a PID controller $\left(K_{p}, K_{i}, K_{d}\right)$ are carefully obtained experimentally or though a process reaction curve [17]. PID controllers work extremely fast, accurate and stable given that the physical plant can be modeled with a set of linear differential equations.

Fuzzy logic controller was chosen in this work to execute an embedded-based air pressure control system. Although PID controllers excel at being accurate and stable, they work well when a complete knowledge about the physical plant is known. Choosing the proper PID parameters requires expert knowledge about the plant. Fuzzy logic controllers, on the other hand, are more robust and often used when complete knowledge about the plant is not known or can change dynamically which is the case of air pressure control system. Factors such as air leakage of the solenoid valves or leakage due to non-existence of perfectly air-tight system would cause the pressure control system to change dynamically. Program 1 shows the simplified algorithm for the fuzzy logic control of air pressure.

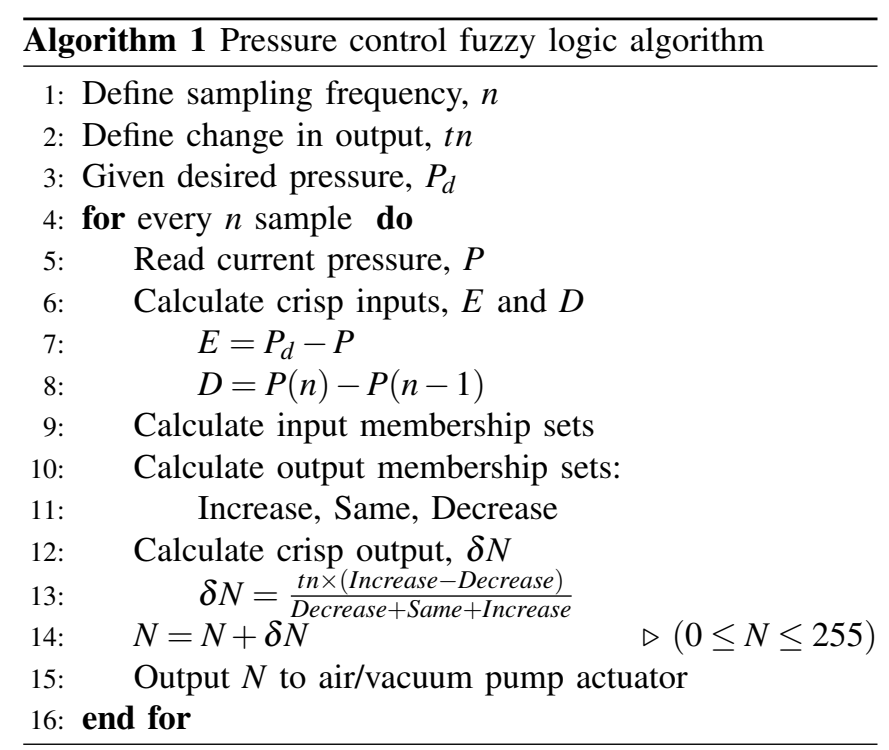

The controller has two crisp inputs, $E$ and $D$. The error signal, $E$, is given by the difference in the desired and current pressure while $D$ is given by difference between the pressure at the current and previous time-step. Six input membership sets are produced from the crisp inputs, which are Slow, Fast, Ok, Down, Up and Constant. Slow, Fast and Ok input membership sets are obtained from $D$ while Down, Up and Constant input membership sets are obtained from $E$. Given these input membership sets. three output membership sets which are Increase, Same, Decrease are produced based on logic functions. The output, $N$, of the software controller is sent to the hardware controller which switches its output pins between $5 \mathrm{~V}$ and $0 \mathrm{~V}$ to create a PWM signal for controlling the speed of the air pump. PWM works because the motor in the air and vacuum pumps have rotational inertia that filters 
out the fast switching. Therefore, the rotational inertia of the DC motor inside the pumps smooths out the rapid pulses provided by PWM and the result is a slower axle rotation and consequently, regulating air pressure.

\section{RESULTS}

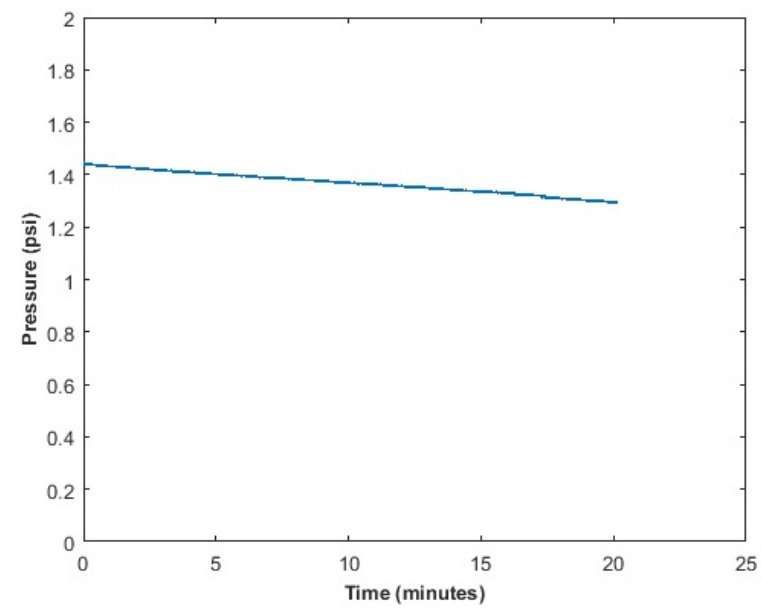

Fig. 4: Air pressure in rubber actuator as a function of time when solenoid valve was in the holding (OFF) state and data collected for 20 minutes.

Before undertaking experiments involving control, the performance of the solenoid valves in the holding (OFF) state was assessed. This was done by inflating a silicone rubber actuator to $\approx 1.4 \mathrm{psi}$ after which the solenoid valves was left in the holding state, air pressure reading was then recorded for 20 minutes, the graph depicting the result is shown in Fig. 4. It can be observed that a pressure drop of 0.12 psi is obtained after 20 minutes - this indicates the suitability of the valves so that they are well suited to carry out pressure control. This result also indicates diffusion of air molecules that occurs through silicone rubber showing that it is not perfectly air tight even when the system is perfectly closed. Rubber actuators are not perfectly air-tight - air molecules slowly diffuse through microscopic pores of stretched rubber when filled with compressed air.

Fig. 5a and 5b show graphs of the performance of the fuzzy logic algorithm in regulating air pressure inside a rigidbodied container between $0-5$ psi. From both graphs, it can be seen that the fuzzy logic controller performed well in reaching and maintaining the desired pressure for varying amounts of time duration.

Fig. 6a and $6 \mathrm{~b}$ show graphs representing the performance of the fuzzy logic controller in regulating air pressure inside a wedged shaped soft robot between $0-1.5$ psi. It can be observed that it takes a longer time period for soft robots to attain a desired pressure compared to rigid wall container. The fuzzy logic controller is able to keep fluctuations within about \pm 0.3 psi from the desired positive pressure.

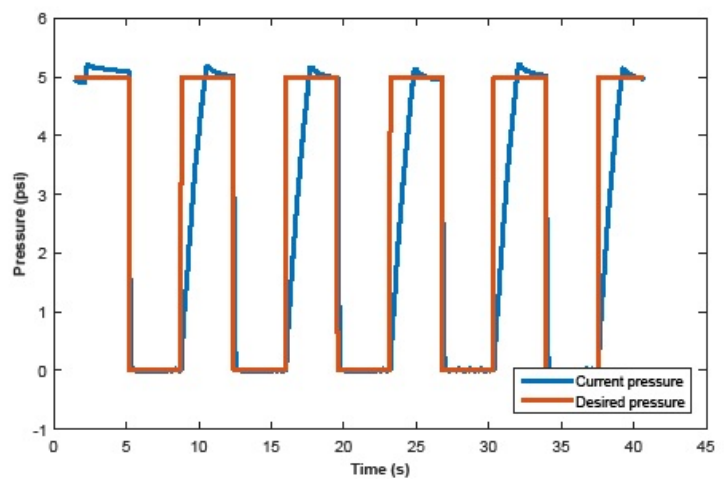

(a)

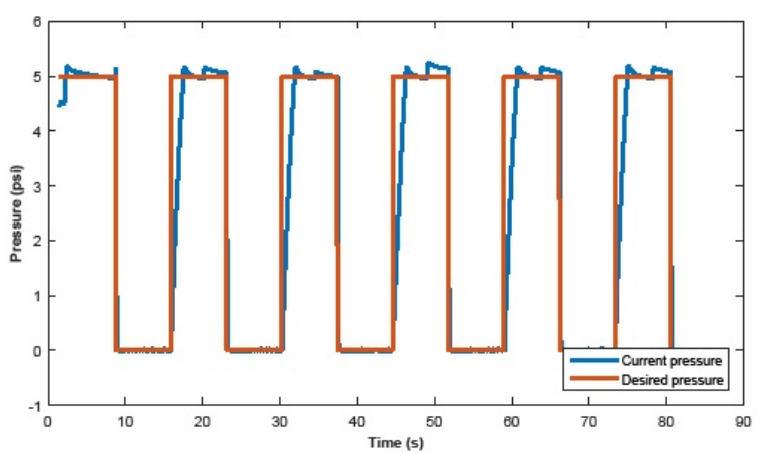

(b)

Fig. 5: Air pressure as a function of time showing performance of the fuzzy logic controller in regulating air pressure between $0-5 \mathrm{psi}$ for a rigid-bodied plastic container. (a) Time to maintain the desired pressure is $4 \mathrm{~s}$ (b) Time to maintain the desired pressure is $8 \mathrm{~s}$.

The embedded fuzzy logic controller was also used to regulate negative air pressure inside silicone rubber actuator by replacing the air pump with a vacuum pump, negative air pressure was regulated between 0 to -5 psi. From Fig. 7, it can be observed that the controller was able to attain the desired negative pressure in $23.5 \mathrm{~s}$. Compared to regulating positive air pressure, a faster rate of attaining the desired negative pressure and lesser degree of fluctuations (within $\pm 0.05 \mathrm{psi}$ ) is observed.

\section{DISCUSSION}

Low pressure values were obtained because a soft elastic body increases in area when inflated which leads to a drop in pressure. Therefore, a soft, highly extensible actuator having the same area with a rigid container produces a relatively lower air pressure reading compared with the rigid container. Fig. 5a and $6 \mathrm{a}$ show graphs of a rigid container regulated from 0psi to 5.0psi and a soft robot inflated and regulated from 0 psi to 1.5 psi using the fuzzy logic controller. From the graphs of the embedded fuzzy logic controller with silicone rubber actuators, it was observed that the pressure rating is lower compared to what would be obtained when filling a rigid wall container with air. This is because as a stretchable 


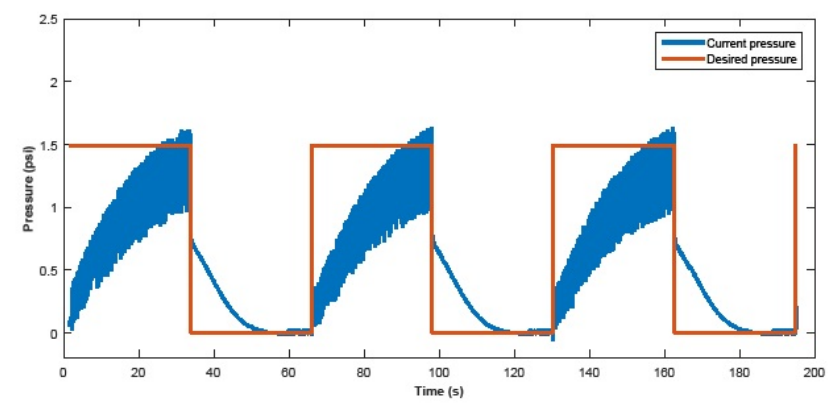

(a)

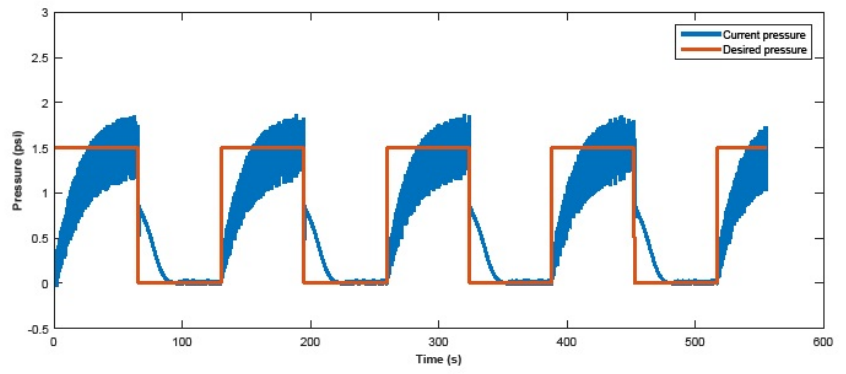

(b)

Fig. 6: Air pressure as a function of time showing performance of the fuzzy logic controller in regulating air pressure between $0-1.5$ psi for a soft, wedge-shaped robot. (a) Time to maintain the desired pressure is $30 \mathrm{~s}$. (b) Time to maintain the desired pressure is $65 \mathrm{~s}$

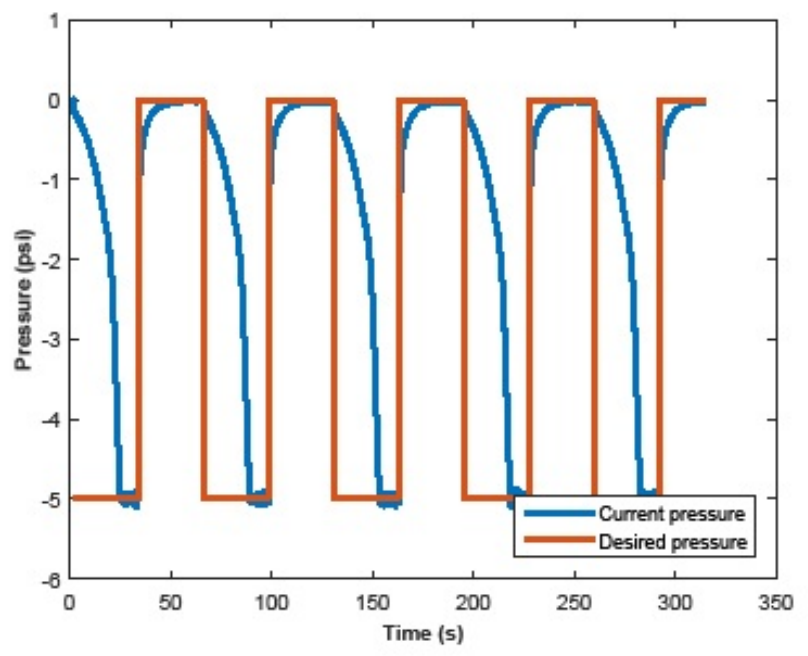

Fig. 7: Air pressure as a function of time showing performance of the fuzzy logic controller in regulating negative air pressure from 0 to $-5 \mathrm{psi}$ for a soft wedged shaped robot. Time to maintain the desired pressure is at a cycle rate of $33 \mathrm{~s}$. material is being inflated, the structure is undergoing a volume expansion as a result of deformation of its structure. This volume expansion will also be reflected by an increase in surface area. As a result of this, the pressure reading will be reduced because of the relationship: $P=\frac{F}{A}$ where $\mathrm{P}=$ Pressure $; \mathrm{F}=$ Force and $\mathrm{A}=$ Area. Since the area is increasing, while the Force remains the same, pressure consequently has to be low. This observation is also reflected in the actuation time of soft actuators to be considerably slower given the hardware system. It was also observed that fluctuations in positive air pressure readings is obtained, this due to the nature of the soft, elastic body being actuated as rigid wall containers do not show these fluctuations under the same fuzzy logic controller. Conversely, negative air pressure results indicate a high operating vacuum pressure. This is due to the buckling phenomenon of rubbers when vacuum operated resulting from volume reduction which consequently increases the operating vacuum pressure.

Controllers for pneumatic soft actuators need to involve pressurization and de-pressurization system as well as suitable control which has been presented using an electropneumatic setup. Though the use of fluidic drive pistoncylinder arrangement rather than solenoid valves would provide a more analog control of pressure by enabling precise analogue control of airflow into and out of actuators, the results that have been presented in this work indicate the viability of the hardware components together with the software control system in actuating and controlling positive and negative air pressure within silicone-based soft robots. This controller system can be used for a variety of pneumatic soft robots to achieve bending, rotary and contraction motions. Results also show the suitability and performance of the hardware and software system in actuating soft robots operating at low pressures, typically between -5 to $+5 \mathrm{psi}$. This pressure controller system can be modified to work with high pressure requirements such as an air compressor-air receiver arrangement which would be a high pressure system because the compressor and air tank are heavy duty accommodating more than 200psi.

In general, the more ambitious the requirements of control are, the more bulky the system will be and system compactness consequently reduces as the size of valves will affect compactness and portability requirements. This also applies to the time taken for the software control to run given the clock speed. The extent of compactness is also reliant on the degree of integration of control components such as the sensors and valves. For a 2 or more actuator system such as robot requiring a multichannel pressure regulation, the number of transistors, pressure sensors and solenoid valves would increase accordingly. The compactness also depends on the tubing and cables needed for operation. Other pneumatic soft actuators such as McKibbean muscles can be controlled with this system, whereby each muscle will be actuated by 2 solenoid valves. This will also be required in 
cases requiring an antagonist setup or multi-finger actuators where each of the fingers requires a pair of solenoid valves for grasping tasks. For high pressure systems, the air source may be excluded from the control so as to reduce size and weight. This would allow for the use of an air-tank containing compressed air as the pressure source. An airtank arrangement may be superior in terms of speed and high pressure requirements but comes at the expense of being bulky and noisy. The solution employed in this work with the use of DC air and vacuum pumps allow for compact, light-weight, portable and a less noisy $(<67 \mathrm{~dB})$ pneumatic system.

Fuzzy logic control strategy presents a promising approach since developed model parameters may not work in practice owing to a number of factors such as change in material properties as inflation-deflation cycles increase. It is also well known that the control of systems that use pneumatic air as actuation power is difficult due to the non linearity of air compressibility, identifying the correct system model and unknown disturbances. The large deformation experienced by pneumatic soft robots means that air pressures set for a certain equilibrium position will depend on its current position. Therefore, air pressure will vary as position varies and vice versa. As a result of these highlighted reasons, the fuzzy logic controller would provide a suitable control since it extrapolates from what it knows in the event of the unknown given unforeseen and unpredictable situations that have been described.

\section{CONCLUSION}

The focus of this work is a software controller based on fuzzy logic to regulate positive and negative internal air pressure inside silicone rubber based actuators. The system is modular in terms of mechanical modularity, supply of compressed air, electrical power supply and system integration. The control algorithm of this system can be easily modified to cover a wide range of movements such as for regulating the required positive and negative air pressure to attain a set bending or rotary angle or contraction amount for a given soft robot. The valve network involving a multichannel arrangement of valves is advantageous in its ability to control many outputs in parallel from a single pressure input.

\section{REFERENCES}

[1] R. V. Martinez, C. R. Fish, X. Chen, and G. M. Whitesides, "Elastomeric origami: Programmable paper-elastomer composites as pneumatic actuators," Advanced Functional Materials, vol. 22, no. 7, pp. 1376-1384, 2012.

[2] V. Oguntosin, W. Harwin, S. Kawamura, S. Nasuto, and Y. Hayashi, "Development of a wearable assistive soft robotic device for elbow rehabilitation," in 2015 IEEE International Conference on Rehabilitation Robotics (ICORR), Aug 2015, pp. 747-752.

[3] A. Wilkening, D. Baiden, and O. Ivlev, "Assistive control of motion therapy devices based on pneumatic soft-actuators with rotary elastic chambers," in 2011 IEEE International Conference on Rehabilitation Robotics, June 2011, pp. 1-6.
[4] K. Hye-Jong, Y. Tanaka, A. Kawamura, S. Kawamura, and Y. Nishioka "Development of an inflatable robotic arm system controlled by a joystick," in 24th IEEE International Symposium on Robot and Human Interactive Communication (RO-MAN), Aug 2015, pp. 664-669.

[5] Y. She, C. Li, J. Cleary, and H. Su, "Design and fabrication of a soft robotic hand with embedded actuators and sensors," Journal of Mechanisms and Robotics, vol. 7, no. 2, pp. 1-9, 2015.

[6] I. Vanderniepen, R. Van Ham, J. Naudet, M. Van Damme, B. Vanderborght, R. Versluys, and D. Lefeber, "Novel compliant actuator for safe and ergonomic rehabilitation robots - design of a powered elbow orthosis," in 2007 IEEE 10th International Conference on Rehabilitation Robotics, June 2007, pp. 790-797.

[7] D. Kim, "Compliant motion control for a compliant rehabilitation system," in 2015 IEEE International Conference on Rehabilitation Robotics (ICORR), Aug 2015, pp. 422-427.

[8] F. Ilievski, D. Mazzeo, Aaron, R. F. Shepherd, X. Chen, and G. M. Whitesides, "Soft robotics for chemists," Angewandte Chemie International Edition, vol. 50, no. 8, pp. 1890-1895, 2011.

[9] R. Shepherd, F. Ilievski, W. Choi, S. Morin, A. Stokes, A. Mazzeo, X. Chen, M. Wang, and G. Whitesides, "Multi-gait soft robot," PNAS, vol. 108, no. 50, p. 2040020403, 2011.

[10] A. Marchese, K. Komorowski, C. Onal, and D. Rus, "Design and control of a soft and continuously deformable $2 \mathrm{~d}$ robotic manipulation system," in 2014 IEEE International Conference on Robotics and Automation (ICRA), May 2014, pp. 2189-2196.

[11] M. Mihajlov, M. Hubner, O. Ivlev, and A. Graser, "Modeling and control of fluidic robotic joints with natural compliance," in IEEE International Conference on Control Applications Computer Aided Control System Design, Oct 2006, pp. 2498-2503.

[12] M. Jordan, D. Pietrusky, M. Mihajlov, and O. Ivlev, "Precise position and trajectory control of pneumatic soft-actuators for assistance robots and motion therapy devices," in IEEE International Conference on Rehabilitation Robotics (ICORR), June 2009, pp. 663-668.

[13] A. Wilkening, M. Mihajlov, and O. Ivlev, "Model-based pressure and torque control for innovative pneumatic soft-actuators," in Proceedings of International Conference on Fluid Power, 2010, pp. 291-302.

[14] P. Polygerinos, Z. Wang, J. T. B. Overvelde, K. C. Galloway, R. J. Wood, K. Bertoldi, and C. J. Walsh, "Modeling of soft fiber-reinforced bending actuators," IEEE Transactions on Robotics, vol. 31, no. 3, pp 778-789, June 2015.

[15] B. Mosadegh, A. D. Mazzeo, R. F. Shepherd, S. A. Morin, U. Gupta, I. Z. Sani, D. Lai, S. Takayama, and G. M. Whitesides, "Control of soft machines using actuators operated by a braille display," Lab Chip, vol. 14, pp. 189-199, 2014.

[16] L. Zadeh, "Fuzzy sets and systems," International Journal of General Systems, vol. 17, no. 2, pp. 129-138, 1990.

[17] J. Valvano, in Embedded Microcomputer Systems: Real Time Interfacing, 3rd ed. Cengage Learning-Engineering, 2011.

[18] K. Hirota and M. Sugeno, Industrial Applications of Fuzzy Technology in the World, 1995, vol. 2.

[19] D. Pelusi, R. Mascella, L. Tallini, L. Vazquez, and D. Diaz, "Control of drum boiler dynamics via an optimized fuzzy controller," International Journal of Simulation: Systems, Science and Technology, vol. 17, no. 33, p. 17, 2016.

[20] D. Pelusi, "Pid and intelligent controllers for optimal timing performances of industrial actuators," International Journal of Simulation: Systems, Science and Technology, vol. 13, no. 2, p. 17, 2012.

[21] V. W. Oguntosin, S. J. Nasuto, and Y. Hayashi, "A compact low-cost electronic hardware design for actuating soft robots," pp. 242-247, March 2015.

[22] C. J. Nycz, T. Btzer, O. Lambercy, J. Arata, G. S. Fischer, and R. Gassert, "Design and characterization of a lightweight and fully portable remote actuation system for use with a hand exoskeleton," IEEE Robotics and Automation Letters, vol. 1, no. 2, pp. 976-983, July 2016. 